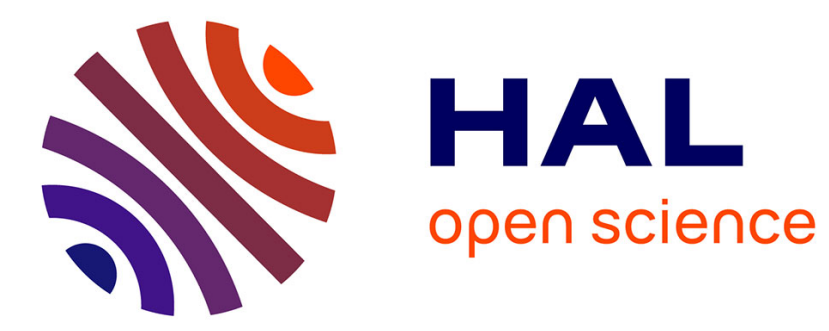

\title{
Essais de tubes souples pour hautes pressions jusqu'à 25 kilobars
}

\author{
J.M. Besson, J.P. Pinceaux
}

\section{To cite this version:}

J.M. Besson, J.P. Pinceaux. Essais de tubes souples pour hautes pressions jusqu'à 25 kilobars. Revue de Physique Appliquée, 1972, 7 (2), pp.137-138. 10.1051/rphysap:0197200702013700 . jpa-00243605

\section{HAL Id: jpa-00243605 https://hal.science/jpa-00243605}

Submitted on 1 Jan 1972

HAL is a multi-disciplinary open access archive for the deposit and dissemination of scientific research documents, whether they are published or not. The documents may come from teaching and research institutions in France or abroad, or from public or private research centers.
L'archive ouverte pluridisciplinaire HAL, est destinée au dépôt et à la diffusion de documents scientifiques de niveau recherche, publiés ou non, émanant des établissements d'enseignement et de recherche français ou étrangers, des laboratoires publics ou privés. 


\title{
ESSAIS DE TUBES SOUPLES POUR HAUTES PRESSIONS JUSQU'A 25 kilobars
}

\author{
J. M. BESSON, J. P. PINCEAUX \\ Laboratoire de Physique des Solides de Paris VIe Tour 13-E2, 9, Quai Saint-Bernard, Paris Ve
}

(Reçu le 27 janvier 1972)

Dans de nombreux cas, il est nécessaire, aux moyennes pressions (10 à 30 kilobars) de disposer de tubes souples pour transmettre la pression. A notre connaissance, aucun progrès n'a été rapporté dans ce domaine depuis la mise au point de tubes d'acier inoxydable [1], [2] étirés, utilisables jusque vers 18 kilobars seulement. Nous donnons ici les performances de tubes capil- pentane-isopentane (2-méthylbutane) et les essais rapportés ici ont été faits à température ambiante.

A l'origine, le but de cette série d'essais était de trouver la limite de rupture des tubes. Il nous a été impossible, pour l'instant, d'obtenir la rupture, l'appareillage actuel n'étant pas conçu pour fonctionner très au-dessus de 25 kilobars.

\section{TABLEAU}

\begin{tabular}{|c|c|c|c|c|c|}
\hline $\begin{array}{l}\text { Numéro } \\
\text { du tube }\end{array}$ & \multicolumn{2}{|c|}{ Diamètre mm } & \multicolumn{3}{|c|}{$\begin{array}{l}\text { Pression } \\
\text { maximum }\end{array}$} \\
\hline - & - & - & - & - & - \\
\hline 1 & 3,1 & 0,4 & $\begin{array}{l}\text { Soudure sous argon vieillie } \\
\text { à } 480^{\circ} \mathrm{C}\end{array}$ & 25 & pas d'explosion \\
\hline 2 & 1,6 & 0,3 & $\begin{array}{l}\text { Soudure sous argon vieillie } \\
\text { à } 480^{\circ} \mathrm{C}\end{array}$ & 22 & explosion à la soudure \\
\hline 3 & 1,6 & 0,3 & $\begin{array}{l}\text { Vissé-soudé sur l'obtura- } \\
\text { teur d'une chambre }\end{array}$ & 24 & pas d'explosion \\
\hline 4 & 1,6 & 0,3 & $\begin{array}{l}\text { Vissé-soudé sur l'obtura- } \\
\text { teur d'une chambre }\end{array}$ & 25,5 & pas d'explosion \\
\hline
\end{tabular}

laires d'acier à durcissement structural (maraging) Marphy — de diamètre 3,2 $\mathrm{mm}$ ext, $0,4 \mathrm{~mm}$ int.; et $1,6 \mathrm{~mm}$ ext., $0,3 \mathrm{~mm}$ int. Ces tubes sont fabriqués par étirage à froid au mandrin.

Les tubes essayés sont vissés et soudés à l'alliage étain-plomb ordinaire sur l'obturateur inférieur d'une chambre de compression simple 28 kilobars d'acier Marval 18 à âme autofrettée. L'autre extrémité est soit soudée-vissée sur l'obturateur d'une cellule simple 26 kilobars en Marval 18, soit simplement obturée par soudure à l'arc sous argon et vieillissement (maraging) du point de soudure à $480^{\circ} \mathrm{C}$. La mesure de la pression est faite par une jauge à la manganine calibrée logée dans la cellule à l'autre bout du tube, ce quı permet de vérifier que le liquide transmetteur est assez fluide pour passer dans le capillaire (essais 3 et 4). Pour les essais 1 et 2 , la pression a été évaluée par la mesure de la pression dans la chambre de compression primaire. Le transmetteur de pression est soit du pentane soit le mélange $50 \% 50 \%$
Un point doit être noté : c'est la possibilité de soudure autogène sur les aciers Maraging [3]. Après recuit à $480^{\circ} \mathrm{C}$ la tenue aux points de soudure peut atteindre 25 kilobars (essai $n^{\circ} 1$ ). Nous n'avons pas encore fait d'essais de soudure directe obturateurtube ou de soudures bout à bout mais il est probable que ces montages sont possibles. La rupture explosive à l'essai no 2 s'est produite à 22 kilobars sur une boursouflure apparente du point de soudure, il apparaît donc que les capillaires souples d'acier Marphy sont capables de transmettre la pression jusqu'à plus de 25 kilobars ce qui est un progrès significatif par rapport aux tubes d'acier inoxydable (environ 18 kilobars). Comme ces derniers, les tubes de Marphy tiennent à des pressions intérieures supérieures à la limite élastique théorique du matériau massif (dureté RC 50 à 55). Ceci est certainement à relier au procédé de fabrication (étirage à froid) et à la possibilité d'autofrettage.

Nous remercions ici M. Cazin (Société Creusot- 
Loire-Aciéries d'Imphy) pour la fabrication des tubes 91-Orsay) qui est à l'origine de l'utilisation de ces d'essai et tout particulièrement M. Jérôme (Labo- aciers, qui a fait étirer les capillaires de $3 \mathrm{~mm}$ et nous ratoire de Physique des Solides, Faculté des Sciences, les a fournis.

\section{Bibliographie}

[1] Paul (W.), Warschauer (D. M.), Rev. Sci. Instr., $1956,27,418$.

[2] Warschauer (D. M.), Paul (W.), Rev. Sci. Instr., 1958, 29, 675.

[3] LANG (F. H.), KenYON (N.), Welding of Maraging Steels-WRC Bulletin, 1971, 159, 1. 